\title{
Los Break-in parties en América Latina: ¿éxito o fracaso?
}

\author{
The Break-in parties in Latin America: \\ success or failure?
}

\section{Thomas Kestler \\ Silvana Krause Juan Bautista Lucca}

\section{Resumen}

En esta nota de pesquisa se da cuenta del desarrollo teórico y el diseño metodológico del proyecto de investigación "Los Break-in parties en América Latina: ¿éxito o fracaso?”. La propuesta general del proyecto es: analizar las características de estos partidos capaces de "irrumpir" en el sistema de partidos; revelar cómo las condiciones estructurales del momento de la génesis inciden en el desarrollo del partido "hacia adentro" y "hacia afuera"; y establecer una explicación que vincule estos tipos de partidos, su origen y desarrollo, con su éxito o su fracaso posterior. Con base en estos objetivos, en esta nota de pesquisa se llevará a cabo: primero, una revisión bibliográfica sobre el surgimiento de nuevos partidos; segundo, una problematización sobre qué es lo "novedoso" de estas organizaciones; y tercero, una exposición de las principales decisiones teórico y metodológicas para analizar los Break-in parties.

\section{Palabras clave}

Break-in Parties; América Latina; Nuevos Partidos; Desafío al Sistema de Partidos; Composición de las Elites; Consolidación Democrática.

\section{Abstract}

This research note outlines the theoretical development and methodological design of the research project "Break-in parties in Latin America: Success or Failure?". The aim of the project is to analyze the characteristics of parties that manage to break into the party system - to clarify how structural conditions at the moment of genesis affect the "inward" and "outward" development of these parties and to explain how its origin and development are linked to their subsequent success or failure. To expose these objectives, in this research-note we procede as follows: first, we offer a literature review on the emergence of new parties; second, we discuss what is "new" in these kinds of organizations; and third, we explain our theoretical and methodological approach to analyze the break-in parties in Latin America.

\section{Keywords}

Break-in Parties; Latin America; New Political Parties; Challenger Parties and Party System; Political Behavior of Party's Elite; Democratic Consolidation. 


\section{Introducción}

La presente nota de pesquisa da cuenta del estado actual del proyecto de investigación "Los Break-in parties en América Latina: ¿éxito o fracaso?" que se lleva a cabo desde el año 2012 entre investigadores de universidades latinoamericanas (Universidade Federal do Rio Grande do Sul en Brasil y Universidad Nacional de Rosario en Argentina) y europeas (Julius-MaximiliansUniversität Würzburg en Alemania y Universität Salzburg en Austria), con el objetivo de estudiar el surgimiento de nuevos partidos capaces de modificar los patrones de representación dentro de los sistemas de partidos en América del Sur.

La propuesta general del proyecto $^{1}$ es analizar, en primer lugar, las características de estos partidos capaces de "irrumpir" (Break-in parties) en el sistema de partidos; en segundo lugar, revelar en qué medida las condiciones estructurales del momento de la génesis incide en el desarrollo del partido "hacia adentro" y "hacia afuera"; y en tercer lugar, poder establecer una explicación que vincule estos tipos de partidos, su origen y desarrollo, con su éxito o su fracaso (KESTLER, KRAUSE y LUCCA, 2013).

Con base en estos objetivos, esta nota de pesquisa habrá de llevar a cabo, en primer lugar, una revisión bibliográfica sobre cómo se explicó el surgimiento de nuevos partidos; en segundo lugar, problematizar acerca de lo "novedoso" en estas organizaciones; $y$, por último, presentar las principales decisiones teórico metodológicas del proyecto para abordar el surgimiento, éxito o fracaso de los Break-in Parties.

¿Cómo surgen nuevos partidos?

La literatura sobre partidos políticos que estudia el origen de nuevos partidos es amplia y profusa, motivo por el cual a manera de ejercicio analítico, esta será ordenada con base en tres componentes explicativos:

a) Cambios socio-estructurales: fracturas sociales o clivajes, crisis, value changes, coyunturas y oportunidades históricas.

b) Condiciones institucionales: sistemas electorales, primacía de la arena presidencial o parlamentaria, legislación partidaria, etc.

c) Dinámica de los actores: cálculo estratégico de las élites, personalización y tendencia al partido catch-all.

Claramente al utilizar este criterio clasificatorio, existirán perspectivas que complementan varios de estos elementos, o que difícilmente sean clasificables en uno u otro vértice, sin embargo, este ordenamiento de la literatura no supone necesariamente que estos sean compartimentos estancos.

En cuanto a la perspectiva que se focaliza primordialmente en los cambios estructurales, uno de los primer intentos por abordar el aspecto del origen de los

${ }^{1}$ Este proyecto contó con el apoyo financiero para misiones científicas de la Coordenação Aperfeiçoamento do Pessoal de Nível Superior-CAPES (Brasil) y el Deutsche Akademische Austauschdienst-DAAD (Alemania, Brasil y Argentina). 
partidos políticos es el de Maurice Duverger (1995, p. 15), quien diferenció los partidos de "origen interno" de aquellos que provienen de un "origen externo". De esta forma, es posible encontrar "partidos de cuadros" o de "notables", que se conforman por parlamentarios y comités electorales con la finalidad de organizar la disputa electoral; y también "partidos de masas" que se organizan a partir de grupos sociales excluidos de la vida política parlamentaria (DUVERGER, 1995, p. 26-27 e 55). Aunque la tipología de Duverger no presenta claramente una propuesta explícita que permita entender el origen de nuevos partidos, es posible pensar que, para caracterizar los "partidos de masas", parte de la premisa de que existió un proceso de cambio en las estructuras sociales que fomentó la incorporación e integración de nuevos actores a la política, y por ende dio lugar a un nuevo tipo de partidos.

Ahora bien, es importante señalar que el vínculo entre el "lugar de origen" y el cambio socio estructural observado por Duverger, no es suficiente para entender el fenómeno de la formación de nuevos partidos en contextos de creciente diversidad y complejidad política en las democracias contemporáneas. Son innumerables, por ejemplo, los grupos de insiders - elites políticas que ya se encuentran integradas en el sistema político - que aprovechan la oportunidad para crear nuevos partidos. Muchas veces, esta iniciativa es motivada o presionada por una crisis de legitimidad y de representación imperante, y no necesariamente encuentra su sustento en la búsqueda de integración de clases o grupos sociales que reivindican la participación e integración en el sistema; es decir, son creados a partir de enfrentamientos por crear nuevas "banderas" o siglas partidarias. No se trata entonces de nuevos partidos de origen externo, ni partidos de la elite, ya que estas nuevas configuraciones cristalizan fundamentalmente cambios socio culturales asociados a nuevas formas de comportamiento y valores políticos, que llevan por ende a nuevas demandas programáticas (MIRAGLIOTTA, 2012) que no se contemplan satisfactoriamente en los partidos tradicionales ya establecidos en el juego político (SIKK, 2012).

Otro esfuerzo por entender el origen partidario, tomando justamente la complejidad y el cambio en la estructura social como generador de nuevas leyendas partidarias, es el que proponen Joseph LaPalombara y Myron Weiner (1972), quienes elaboran un modelo explicativo tomando en cuenta tres tipos de crisis: de legitimidad, de integración y de participación. Inclusive, en un esfuerzo teórico complementario a esta perspectiva, otros autores como Kenneth Janda (1980) vincularon el origen y desarrollo de los partidos con las coyunturas críticas en las que se desencadenan la fusión o escisión de partidos preexistentes, así como también el surgimiento de nuevos liderazgos en torno de los cuales se forman los partidos.

Esta perspectiva que se concentra en las transformaciones o cambios estructurales de la sociedad como el generador de nuevos intereses y actores a representar por nuevos partidos, puede encontrarse también en la propuesta de 
Seymour Lipset y Stein Rokkan (1967) y su concepto de "clivajes" y la "hipótesis del congelamiento". Allí, los autores sostienen que las leyendas partidarias nacen producto de las fracturas que genera la configuración del estado nación y la revolución industrial, y se consolidan en el tiempo en tanto y en cuanto estos sismas sociales se mantengan. Inclusive, extendiendo la teoría de Lipset y Rokkan tiempo después, Herbert Kitschelt (1997) identifica como factor central para comprender a la fundación de nuevas siglas partidarias al cambio en las estructuras de clivajes como consecuencia de la aparición de nuevos grupos sociales y orientaciones normativas (value change).

Sin embargo, para la misma época en que surgieron estas miradas centradas en las fracturas sociales, Otto Kircheimer (1972) observa cómo las identidades partidarias sustentadas en clivajes socio-económicos se volvieron fluidas y gelatinosas, dando origen a la transformación de los "partidos de masa" en "partidos catch-all" después de la segunda guerra mundial. Este fenómeno parte del supuesto de que el papel central de integración de los grupos sociales en el sistema político ya fue cumplido por los partidos de "origen externo" y carecían de razón de ser en este nuevo contexto (LAPALOMBARA, 2007; LAPALOMBARA y WEINER, 1972; BOLLEYER, 2012).

A pesar que la preocupación de Kircheimer no sea exactamente la de entender por qué surgen nuevos partidos, es posible reconocer que su perspectiva abre nuevos horizontes para la comprensión de este fenómeno, especialmente porque coloca el acento en observar el perfil y accionar de los actores inmersos en un determinado juego político. El autor desacopla, de esta forma, el contexto originario de la función de la organización partidaria, ya que considera que en las democracias contemporáneas la formación de nuevas leyendas no encuentra su impulso en la integración al sistema político, sino más bien por estrategias políticas de las élites orientadas por factores coyunturales el mercado político.

Esta afirmación de Kircheimer en la década de 1960 se encuentra fuertemente emparentada con visiones posteriores como las de Ángelo Panebianco (2005) y Peter Mair (1994). La perspectiva organizacional de Panebianco contribuye para destacar las diferentes estrategias que llevan adelante los actores políticos en contextos de formación de organizaciones partidarias (penetración y difusión); mientras que Mair, señala que el disparador para la creación de nuevas leyendas partidarias estaría vinculado con el cálculo estratégico que implica alcanzar la "maquina" de gobierno, y no con las "incubadoras" partidarias vinculadas con la competencia electoral y los clivajes sociales. En síntesis, es posible señalar que esta perspectiva centrada en los actores fundamenta el origen partidario en el cálculo racional, en el marco de un mercado electoral altamente competitivo e inestable, en el que se sopesan los costos y beneficios de entrar a la arena política con un nuevo partido (TAVITS, 2008).

Aunque muchos estudios sobre los orígenes de los partidos tengan en cuenta explicaciones múltiples (VAN COTT, 2003), es posible reconocer un 
tercer enfoque que toma en cuenta las instituciones como variable central de su argumento. Por ejemplo, Robert Harmel y John Robertson (1985), inspirados tal vez en la perspectiva de Duverger, observan que los sistemas proporcionales incentivan la representación de un número mayor de partidos, lo que induciría a la formación de nuevos partidos; sin embargo no explicitan de forma clara cómo actúa este incentivo institucional en la génesis misma de las organizaciones partidarias. Otros esfuerzos, como los de Joseph Willey (1998), al dar cuenta en qué medida las variables institucionales como el tamaño del distrito, la configuración federal-unitaria y la forma de gobierno impactan en las chances de los nuevos partidos para alcanzar espacios de representación política, focalizan sus esfuerzos en las claves del éxito de estos nuevos partidos, pero no necesariamente en cómo estos factores institucionales inciden en su génesis. Inclusive, autores como Simon Hug (2000), demuestran que los factores institucionales como las leyes de creación y registro de los partidos y el establecimiento formal de las formas de financiamiento partidario son condiciones explicativas para el surgimiento de los partidos, pero no de su desarrollo posterior. En síntesis, es posible advertir que el enfoque institucional pone énfasis en los elementos que "habilitan" la incorporación de nuevos actores, pero no explica claramente cómo incide esto en la formación en sí de nuevas organizaciones, sino más bien da cuenta de los factores que intervienen en el éxito o fracaso de los partidos políticos.

\section{¿Cuál es la novedad en los nuevos partidos?}

Más allá de interrogarse cómo surgen los partidos, es necesario poder dar cuenta qué hay de nuevo en estos ya que, como señaló Janda ${ }^{2}$ (apud BARNEA y RAHAT, 2010), muchas veces los nuevos partidos derivan de la extinción o unión de dos o más partidos tradicionales, con lo cual su "novedad" es escasa para el sistema (LÓPEZ, 2005). Este es uno de los interrogantes centrales del presente proyecto, ya que considera que producto del cambio de una matriz estado céntrica a una mercado céntrica, la crisis de representación de los actores colectivos y el cambio en el régimen político que vivió América Latina en las últimas cuatro décadas, existiría una estructura de oportunidades políticas para el surgimiento de

\footnotetext{
2 De acuerdo con la interpretación de Barnea y Rahat sobre la perspectiva de Janda, hay tres posibilidades en este punto: "The first is that, notwithstanding any other criteria for newness, a party that retains its old name is seen in this respect as an old party. The second possibility is that of a party which uses a brand new name, with no trace of the old - possibly in an attempt to dissociate itself from the 'establishment' and market itself as a fresh, new alternative. A third possibilityis that of a party that chooses to adopt a namethat includes traces of the past. In the case of existing parties, this may symbolize a promise to revamp the old party so as to boost its appeal to new groups of voters while preserving its identity in the eyes of its loyal, veteran constituency for example, adding the adjective 'new' to the old party label" (BARNEA y RAHAT, 2010, p. 307).
} 
partidos de nuevo cuño, que en este proyecto caracterizamos como Break-in parties (TARROW, 1997; TILLY, 1984; MEYER y MINKOFF, 2004).

La novedad del surgimiento de estos partidos, es que son capaces de irrumpir en una escena partidaria en transformación, establecer nuevas modalidades de representación política, y a su vez fortalecer la democracia al incorporar nuevos sectores a la participación político partidaria. Para comprender con mayor detalle qué los caracteriza, en este proyecto de investigación se entiende a los Break-in parties como aquellos partidos que logran imponer el carácter de innovador en dos dimensiones de análisis: en el desafío al sistema de partidos y en la composición de las elites.

En la primera dimensión, se parte de la premisa de que en la competencia partidaria, existirían tres situaciones: en primer lugar, aquellos que detentan las posiciones de privilegio (partidos del status quo) dentro del sistema de partidos, y que en virtud de esto, buscan que no se alteren las reglas de juego, los roles, actores e intereses a representar dentro del mismo. En segundo lugar, partidos que buscan alterar las posiciones dentro del sistema político, a través de la movilización popular, y que por ende adquieren el rotulo de anti-status quo; y en tercer lugar, partidos que desafían también al status quo del sistema de partidos, pero arremeten de forma radical contra el sistema político en su conjunto, al buscar alterar las reglas de juego y las posiciones de los actores a través del uso de la violencia física como uno de sus medios de injerencia política, que denominaremos partidos anti sistema ${ }^{4}$.

Ahora bien, estas tres situaciones partidarias poco dicen respecto de la relevancia de los partidos, al decir de Giovanni Sartori (1976), puesto que no todo desafío al status quo es en sí una amenaza para este, ni la presencia de partidos anti sistemas es en sí una amenaza para la democracia, en tanto y en cuanto estos partidos no adquieren una relevancia electoral sustantiva que los coloque como una verdadera amenaza (al status quo y el sistema político). Por tal motivo, en este proyecto la capacidad de ser partidos desafiantes es una relación proporcional entre la condición frente al status quo y su relevancia electoral.

La segunda dimensión para observar la condición de novedoso de los nuevos partidos, relativa a la composición de las élites, toma en cuenta que no todos los partidos cuando surgen incorporan nuevos agentes a la vida partidaria, motivo por el cual habremos de clasificar a los partidos políticos latinoamericanos durante las últimas cuatro décadas observando si su fundación u origen fue de tipo interno o externo según Duverger; es decir, si son partidos cuyas elites ya se encontraban actuando en el sistema partidario (insiders), si son siglas partidarias

\footnotetext{
${ }^{3}$ Es importante señalar que este proyecto no tiene como objetivo analizar estos partidos cuando asumen el poder ejecutivo nacional.

${ }^{4}$ Genéricamente este término es utilizado para partidos con ideales no democráticos, que dan sustento a actividades ilegales o no convencionales (LINZ, 1987; SARTORI, 1976; CAPOCCIA, 2002).
} 
que conjugan viejas y nuevas élites (casos intermedios) o si son partidos que incorporaron nuevas élites en su totalidad al momento de su fundación (outsiders) ${ }^{5}$.

Partiendo de la base de esta distinción analítica, es posible entonces delimitar que los Break-in parties son aquellos partidos que son nuevos tanto en su capacidad para desafiar el status quo como también en incorporar nuevas elites políticas al juego de la competencia partidaria. Por ello, los Break-in parties serían de enorme importancia para la consolidación democrática de los países latinoamericanos en el contexto del cambio del modelo socioeconómico, del régimen político y la crisis de representación política durante la década de 1990, pues serían partidos capaces de movilizar nuevos sectores sociales e incorporar nuevos actores políticos a través de canales electorales, es decir canalizar el conflicto de la incorporación de nuevos actores a la democracia (HIGLEY y GUNTHER, 1992).

Los cuadros 1 e 2 permiten visualizar entonces ambas dimensiones, las diversas variantes que existen al respecto, y cómo será operacionalizada su medición en el presente proyecto.

Cuadro 1 - Desafío al sistema de partidos (Challenger)

\begin{tabular}{|c|c|c|}
\hline Concepto & Definición & Operacionalización \\
\hline $\begin{array}{c}\text { Status Quo } \\
\text { (SQ) }\end{array}$ & $\begin{array}{c}\text { Partidos que no quieren/buscan } \\
\text { alterar las reglas del juego, ni } \\
\text { modifica su papel principal en el } \\
\text { sistema político }\end{array}$ & O x \% de votación \\
\hline $\begin{array}{c}\text { Anti Status Quo } \\
\text { (ASQ) }\end{array}$ & $\begin{array}{c}\text { Partidos que buscan alterar las } \\
\text { posiciones dentro del sistema } \\
\text { político, a través de la movilización } \\
\text { popular (no electoral) }\end{array}$ & $1 \times$ de votación \\
\hline Anti Sistema (AS) & $\begin{array}{c}\text { Partidos que buscan alterar las reglas } \\
\text { de juego, las posiciones dentro del } \\
\text { sistema a través da utilización de } \\
\text { medios violentos }\end{array}$ & $2 \times \%$ de votación \\
\hline
\end{tabular}

* Se seleccionan los casos como SQ, ASQ, AS en el año de la mayor votación conquistada. Fuente: Elaboración propia.

\footnotetext{
5 Miguel Carreras (2012) ofrece una distinción entre "outsiders e insiders", definida en relación con el origen del político en el sistema partidario y su experiencia política previa. Sin embargo, aunque resulta sugerente, nuestra propuesta se focaliza en un criterio clásico y simplificador como el de Duverger, ya que de otra manera implicaría adjetivar el concepto con expresiones como "populistas", "neopopulistas", entre otros, que ganarían en intensidad explicativa pero perderían en extensión comparativa, y por ende en un argumento generalizable.
} 
Cuadro 2 - Composición de las elites (Outsider)

\begin{tabular}{|c|c|c|}
\hline Concepto & Definición & Operacionalización \\
\hline Insider & $\begin{array}{c}\text { Partido cuyas elites ya se encuentran } \\
\text { actuando en el sistema partidario }\end{array}$ & 1 \\
\hline $\begin{array}{c}\text { Caso } \\
\text { Intermedio }\end{array}$ & $\begin{array}{c}\text { Partido cuyas donde se mezclan viejas y } \\
\text { nuevas elites }\end{array}$ & 2 \\
\hline Outsider & $\begin{array}{c}\text { Partidos cuyas elites se originan fuera del } \\
\text { sistema partidario vigente. }\end{array}$ & 0 \\
\hline
\end{tabular}

* Se seleccionan los casos en el momento de de origen.

Fuente: Elaboración propia.

Con base en esta distinción teórico metodológica sobre los Break-in parties en América Latina, la selección de casos a estudiar estuvo orientada por la construcción de la Latin American Break-in parties database. En esta nueva base de datos se toma en cuenta la información vertida entre otros por Dieter Nohlen (2005) y el Political Database of the Americas, para identificar todos los partidos que surgieron y participaron electoralmente en América del Sur entre la década de 1960 y 2000; y dentro de este amplio universo de casos se clasifican los partidos en relación a ambas dimensiones a través de un juicio de expertos.

En cuanto a la primera dimensión de análisis - su calidad de desafiantes se atribuye a los partidos del status quo un valor de 0 , a los partidos con una postura anti-status quo un valor de 1 , y los partidos anti-sistema un valor de 2 , que se multiplica por su desempeño electoral en el año de mayor votación conquistada, para poder establecer en qué medida cada uno de los partidos fue realmente "desafiante", lo que nos permitirá eliminar de la muestra aquellos que no presentan ninguna desafío y por ende novedad al sistema.

En cuando a la segunda dimensión - su calidad de outsider - los partidos formados por insiders se identifican con un valor de 0 , los casos intermedios con un valor de 1 , y los casos de outsiders, con un valor de 2 , de forma tal que se obtenga una clasificación clara de cuales partidos y en qué medida incorporan nuevos actores políticos a la competencia partidaria.

A través del entrecruzamiento de ambas dimensiones obtendremos un panorama detallado de los casos tentativos, de forma tal que en un momento posterior de la investigación se lleve a cabo el estudio de casos en profundidad, para observar a través de la comparación de un "n pequeño" en qué medida este formato partidario se configuró en el plano de la disputa electoral y la organización interna, buscando allí las claves de su éxito o fracaso de cara al nuevo milenio.

\section{¿Cómo estudiar el éxito/fracaso de los Break-in parties en las democracias latinoamericanas?}

La noción de éxito o fracaso partidario, es claramente una definición polisémica y arbitraria dentro de la discusión teórica sobre los partidos políticos 
(HARMEL y ROBERTSON, 1985) especialmente en contextos heterogéneos y complejos como los de América Latina donde pueden identificarse formas parciales de éxito, tales como el crecimiento continuo de la votación electoral, la mera supervivencia del partido a lo largo del tiempo, o la inclusión dentro del sistema de partido como actor relevante, entre otros (LÓPEZ, 2005). Sin embargo, en esta región, gran parte del proceso político se concentra en torno a la disputa electoral de la presidencia, lo que llevaría a comprender de forma simple que el éxito radica en ocupar el cargo de mayor jerarquía electoral, es decir el Poder Ejecutivo.

En contrapartida a la definición previa, el fracaso no se explicaría solamente por no ocupar la presidencia, ya que esta es una situación intermedia, porque el fracaso en sí de un partido consiste en padecer una división significativa del partido que lo debilite internamente y disponer de un carácter electoral marginal. Entonces, el éxito o fracaso de los Break-in parties en este proyecto es la variable dependiente de ciertos factores explicativos que pueden organizarse en torno a dos dimensiones de análisis: el desarrollo partidario "hacia adentro" y el devenir del partido "hacia afuera". En cuanto a la dimensión explicativa del partido "hacia adentro", las variables independientes a tener en cuenta son la cohesión, la inclusión y la posición del partido hacia el status quo; en tanto que en la dimensión "hacia afuera" la principal variable independiente es el crecimiento electoral.

La principal hipótesis de funcionamiento de estas variables, es que los Break-in parties se enfrentan a la complejidad de obtener un equilibrio dinámico entre su cara hacia afuera y adentro, ya que son factores directamente interrelacionados; motivo por el cual es posible identificar un "círculo virtuoso" en el que cuanto mayor es la cantidad de grupos que se conjugan internamente, y más cohesionados estos se encuentran, más amplia es la base de miembros y mayor es el de grupos de votantes que se puede integrar y por ende mayor la posibilidad de tener una relevancia electoral de jerarquía. Sin embargo, también existe un "círculo vicioso" entre estas variables, ya que cuanto mayor es la movilización electoral, la cohesión interna se torna inestable, la inclusión de nuevos actores genera malestar, la posición frente al status quo se tornan diversas y por ende la chance de que ocurran divisiones significativas y que la expresión electoral sea marginal es mayor.

Para facilitar una comparación sistemática, el análisis histórico del desarrollo partidario será complementado por una evaluación de las variables a través de indicadores claramente establecidos. En este sentido, al analizar la cohesión se tomará en cuenta como indicadores la existencia o no de a) consenso ideológico, b) consenso sobre el liderazgo, c) disputas sobre las reglas internas, d) fracturas internas, e) escisiones de peso y f) la existencia de un único núcleo de poder (jerarquía) o varios (estratarquía). Para analizar la inclusión, los indicadores que se tendrán en cuenta son: g) la facilidad/dificultad del acceso de los 
miembros, h) la homogeneidad o heterogeneidad de estos miembros, i) la composición del liderazgo, j) la relevancia de las organizaciones de la sociedad civil, k) expansión o retracción territorial y l) la rotación o no del liderazgo. En cuanto a los indicadores para observar la posición del partido vis a vis el status $q u o$, se observará: m) la posición - moderada o radical - en elecciones presidenciales, n) la política de alianzas pre-electorales y ñ) la política de alianzas legislativas. Por último, al analizar el desempeño electoral, los indicadores a tener en cuenta son: o) la distribución de cargos y votos en el nivel presidencial, p) en el nivel legislativo y q) en el plano subnacional.

Una vez que se establece claramente, desde una perspectiva cualitativa, el desempeño de cada uno de los indicadores para los casos seleccionados, los resultados cualitativos se convierten en datos cuantitativos, con una valoración de 0 y 1 para los indicadores "a" a " $\tilde{n}$ " y de 0 a 2 para los indicadores "o" a "q", de forma tal que se pueda percibir el derrotero de cada una de las variables a lo largo del tiempo, y sea posible observar las cadenas de causalidad que entre estas variables se establecen en relación con la variable dependiente (éxito-fracaso).

\section{Desafíos y expectativas de desarrollo}

Llevar adelante este proyecto de investigación en su segunda fase de avance, presenta varios "nuevos" desafíos a tener en cuenta:

- El desafío teórico: en primer lugar, implica la difícil tarea de poner en diálogo la literatura sobre tipos de partidos, partidos políticos en América Latina, la caracterización de nuevas formas partidarias, de éxito o fracaso partidario, entre otros aspectos, para poder fundamentar la existencia y relevancia de estos partidos con capacidad de "irrumpir" en la representación política trayendo consigo una novedad al sistema. En segundo lugar, trae consigo la necesidad de colocar el acento en la importancia de la temporalidad, y la configuración de estructuras de oportunidades que intervienen como factores explicativos del origen, desarrollo y éxito/fracaso de estos partidos, motivo por el cual no solo conlleva la reflexión en cuanto a las diversas aristas que tiene el neoinstitucionalismo histórico y su poco habitual uso en el estudio sobre los partidos políticos, sino también estar atentos a las críticas que a este enfoque se han realizado. Por último, tratar de develar que el factor organizativo o el desempeño electoral, son el reverso y anverso del éxito/fracaso, implica tener que reforzar la idea de que la vida interna de los partidos y su desarrollo externo es determinante para entender quienes son aquellos que acceden al gobierno de la cosa pública.

- El desafío metodológico: llevar adelante este proyecto implica tener en cuenta las fortalezas y debilidades de la utilización de la metodología comparada con una selección de casos de tipo "n pequeño", que permite testear a fondo una hipótesis explicativa como la que aquí se presenta, sin 
que ello implique establecer un nivel de generalidad y control de las hipótesis de mayor rango. Asimismo, metodológicamente apelar a una estrategia comparada centrada en un área geográfica como la de América del Sur, trae consigo la complejidad de partir de un contexto homogéneo para establecer un mayor contraste entre los casos, pero también poner el énfasis en una estrategia que coloca el acento tanto en la comparación orientadas a variables como hacia casos.

- El desafío empírico: llevar adelante estudios de caso para cada uno de los Break-in parties seleccionados, no solo implica la difícil tarea de tener un conocimiento pormenorizado de los casos, sino también obtener fuentes y datos empíricos fiables, que tornen al caso comparable con los demás. Además, a pesar de una férrea selección de casos con base en criterios comunes, es un claro desafío empírico el encontrar las líneas cualitativas de corte para cada uno de los indicadores planteados, y poder ser "sensibles" a la singularidad del caso, sin perder de vista el enfoque teórico, para que el proyecto no sea más que una yuxtaposición de casos.

\section{Thomas Kestler é professor da Julius-Maximilians-Universität Wuirzburg - Alemania / Universität Salzburg - Austria. E-mail: thomas.kestler@uni-wuerzburg.de}

Silvana Krause é professora na Universidade Federal de Rio Grande do Sul - Brasil. E-mail: krausesilvana@yahoo.com.br

Juan Bautista Lucca é profesor na Universidad Nacional de Rosario / CONICET - Argentina. E-mail: juanlucca@hotmail.com

\section{Referencia}

BARNEA, Shlomit. RAHAT, Gideon. Out with the old, in with the "new": What constitutes a new party? Party Politics, London, v. 17, n. 3, p. 303-320, mar. 2010.

BOLLEYER, Nicole. New party organization in Western Europe: Of party hierarchies, stratarchies and federations. Party Politics, London, v. 18, n. 3, p. 315-336, mar. 2012.

CAPOCCIA, Giovanni. Anti-Sytem Parties: A Conceptual Reassessment. Journal of Theoretical Politics, Thousand Oaks, v. 14, n. 1, p. 9-35, jan. 2002.

CARRERAS, Miguel. The Rise of Outsider in Latin America, 1980-2010: An Institutionalist Perspective. Comparative Political Studies, Washington, v. 45, n. 12, p.1-32, dez. 2012. 
DUVERGER, Maurice. Os partidos políticos. Rio de Janeiro: Zahar Editores, 1995.

HARMEL, Robert; ROBERTSON, John. D. Formation and Success of New Parties: A Cross-National Analysis. International Political Science Review, New York, v. 6, n. 4, p. 501-523, oct. 1985.

HIGLEY, John; GUNTHER, Richard. Elites and democratic consolidation in Latin America and Southern Europe. Cambridge: Cambridge University Press, 1992.

HUG, Simon. Studying the electoral success of New Political Parties: A Methodological Note. Party Politics, London, v. 6, n. 2, p. 187-197, apr. 2000.

JANDA, Kenneth. Political Parties: A Cross-National Survey. London: Free Press, 1980.

KESTLER, Thomas; KRAUSE, Silvana; LUCCA; Juan B. Conflicto y cohesión en el origen de los nuevos partidos en América del Sur. In: Congreso del Consejo Europeo de Investigaciones Sociales sobre América Latina, 7., Oporto, 2013.

KIRCHHEIMER, Otto. The Transformation of the Western European Party. In: LaPAlOMBARA, Joseph; WEINER, Myron. Political Parties and political Development. Princeton, New Jersey: Princeton University Press, 1972. p. 137176.

KITSCHELT, Herbert. European Party Systems: Continuity and Change. In: ROHDES, Martin Rhodes. Developments in West European Politics. Basingstoke: Macmillan, 1997. p. 131-150.

LAPALOMBARA, Joseph. Reflections on political parties and political development, four decades later. Party Politics, London, v. 13, n. 2, p. 141154, apr. 2007.

LAPALOMBARA, Joseph; WEINER, Myron. The Origin and Development of Political Parties. In: LAPALOMBARA, Joseph; WEINER, Myron. Political Parties and political Development. Princeton/New Jersey: Princeton University Press, 1972. p. 3-42.

LIPSET, Seymour Martin; ROKKAN, Stein. Cleavage Structures, Party Systems, and Voter Alignments: An introduction. In: LIPSET, Seymour Martin; ROKKAN, Stein. Party Systems and Voter Alignements: Cross National Perspectives. New York/London: The Free Press, Macmillan Limited, 1967. p. $1-64$.

LINZ, Juan. La quiebra de las democracias. Madrid: Alianza Editorial, 1987.

LÓPEZ, Santiago. Partidos desafiantes en América Latina; representación política y estrategias de competencia de las nuevas oposiciones. Revista de Ciencia Política, Santiago, v. 25, n. 2, p. 37-64, 2005.

MAIR, Peter. Party Organizations: From Civil Society to the State. In: KATZ, Richard S.; MAIR, Peter. How parties organize: change and adaptation in party 
organizations in Western democracies. Londres: Sage Publications, 1994. p. 121.

MEYER, David. S.; MINKOFF, Debra C. Conceptualizing political opportunity. Social forces, Baltimore, v .82, n. 4, p. 1457-1492, jun. 2004.

MIRAGLIOTTA, Narelle. From local to national: Explaining the formation of the Australian Green Party. Party Politics, London, v. 18, n. 3, p. 409-425, mai. 2012.

NOHLEN, Dieter. Elections in the Americas. A Data Handbook. v. 2. South America. New York: Oxford University Press, 2005.

PANEBIANCO, Angelo. Modelos de partido: organização e poder nos partidos políticos. São Paulo: Martins Fontes, 2005.

SARTORI, Giovanni. Party and Party Systems. A Framwork for Analysis. Cambridge: Cambridge University Press, 1976.

SIKK, Allan. Newness as a winning formula for new political parties. Party Politics, London, v. 18, n. 4, p. 465-486, jul. 2012.

TARROW, Sidney. Poder en movimiento. Movimientos sociales, acción colectiva y política de masas en el Estado moderno. España: Editorial Alianza, 1997.

TAVITS, Margit. Party Systems in the Making: The emergence and Success of New Parties in New Democracies. British Journal of Political Science, Cambridge, v. 38, n. 1, p. 113-133, jan. 2008.

TILLY, Charles. Big Structures, large processes, huge comparisions. New York: Russell Sage Fundation, 1984.

WILLEY, Joseph. Institutional Arrangements and the Success of New Parties in Old Democracies. Political Studies, London, v. 46, n. 3, p. 651-668, 1998.

VAN COTT, Donna Lee. From Exclusion to Inclusion. Bolivia's 2002 Elections. Journal of Latin America Studies, London, v. 35, n. 4, p. 751-755, nov. 2003.

Texto recebido em 15 de julho 2013. Aprovado em 17 de julho de 2013. 
